\title{
Enhancing Robustness of Instability Suppression by Means of Multi-Degree-of-Freedom Nonlinear Energy Sinks
}

\author{
Young S. Lee ${ }^{*}$ Alexander F. Vakakis $†$ Lawrence A. Bergman ${ }^{\ddagger}$ \\ D. Michael McFarland § Gaëtan Kerschen ฯ \\ University of Illinois at Urbana-Champaign, Urbana, Illinois, 61801
}

\begin{abstract}
Multi-degree-of-freedom nonlinear energy sinks (MDOF NESs) are utilized to improve robustness of suppression of limit cycle oscillations (LCOs) due to aeroelastic instability. Bifurcation analysis by a numerical continuation technique shows that controlling occurrence of a limit point cycle (LPC or saddle-node) bifurcation point above a Hopf bifurcation point is crucial to enhancing robustness. Not only greatly can MDOF NESs enhance the robustness of suppression against even strong external disturbances, but they can also yield a similar efficiency even with a smaller mass, compared to the SDOF NESs with the same parameter conditions. Nonlinear modal interactions between the aeroelastic modes and the MDOF NES are examined to demonstrate efficiency of the MDOF NES.
\end{abstract}

\section{Nomenclature}

\begin{tabular}{|c|c|c|}
\hline$b$ & $=$ & semichord length, $c / 2$, where $c$ is a chord-length \\
\hline$C ; C_{1}$ & $=$ & $\begin{array}{l}\text { nondimensional coefficients for essentially nonlinear coupling stiffness, } b^{2} k_{s} / m \omega_{\alpha}^{2} \text {; } \\
\text { linear coupling stiffness, } k / m \omega_{\alpha}^{2}\end{array}$ \\
\hline$C_{L, \alpha}$ & $=$ & lift curve slope, $\partial C_{L} /\left.\partial \alpha\right|_{\alpha=0}$ where $C_{L}$ is the lift coefficient \\
\hline$c_{1}, c_{2}$ & $=$ & nonlinear heave and pitch stiffness factors \\
\hline$d ; \delta$ & $=$ & $\begin{array}{l}\text { offset attachment of the NES to the wing, measured from and positive ahead of the elastic } \\
\text { axis (e.a.); nondimensional offset, } d / b\end{array}$ \\
\hline$E^{\text {Input }}$ & $=$ & instantaneous input energy given to the aeroelastic system \\
\hline$E^{\text {Total }}$ & $=$ & instantaneous total energy of the aeroelastic system coupled with an NES \\
\hline$E_{d}^{N E S}$ & $=$ & instantaneous energy dissipation by an NES \\
\hline$E^{y, \alpha, v}$ & $=$ & instantaneous modal energies in heave, pitch, and NES modes, respectively \\
\hline$e ; \gamma$ & $=$ & $\begin{array}{l}\text { location of the aerodynamic center (a.c.) measured from the e.a. (positive ahead of e.a.); } \\
\text { nondimensional parameter, } e / b\end{array}$ \\
\hline$h, \alpha, z$ & $=$ & $\begin{array}{l}\text { heave (positive downward), pitch (positive clockwise), NES (positive downward) } \\
\text { degrees of freedom }\end{array}$ \\
\hline$K_{h}, K_{\alpha}$ & $=$ & coefficients of linear heave and pitch stiffnesses \\
\hline & $=$ & coefficient of linear coupling stiffness \\
\hline$L, M$ & $=$ & $\begin{array}{l}\text { lift and aerodynamic moment acting at the a.c., respectively; the equivalent } \\
\text { aerodynamic forces at the e.a. are } L_{e a}=L \text { and } M_{e a}=M+e L \approx e L \text { for small angles }\end{array}$ \\
\hline$m, I_{\alpha}$ & $=$ & mass of the airfoil and its mass moment of inertia with respect to the e.a. \\
\hline$m_{s}, k_{s}, c_{s}$ & & mass, essentially nonlinear stiffness coefficient, and damping in the NES \\
\hline & & dynamic pressure, $\frac{1}{2} \rho_{\infty} U^{2}$, where $\rho_{\infty}$ is the de \\
\hline
\end{tabular}

\footnotetext{
${ }^{*}$ Visiting Assistant Professor, Department of Aerospace Engineering

†Professor, School of Applied Mathematical and Physical Sciences, National Technical University of Athens, Athens, Greece; also Adjunct Professor, Departments of Mechanical and Industrial Engineering and Aerospace Engineering, UIUC.

${ }^{\ddagger}$ Professor, Department of Aerospace Engineering, Associate Fellow, AIAA

${ }^{\S}$ Research Associate Professor, Department of Aerospace Engineering, Senior Member, AIAA.

IVisiting Scholar, Department of Aerospace Engineering; currently Postdoctoral Researcher, Aerospace and Mechanical Engineering Department (LTAS), Université de Liège, Liège, Belgium.
} 
$r_{\alpha} \quad=$ radius of gyration of the cross section of the wing, $\sqrt{I_{\alpha} /\left(m b^{2}\right)}$

$S \quad=$ planform area of the wing

$S_{\alpha} ; x_{\alpha} \quad=$ mass unbalance in the airfoil, $m x_{c g}$; its nondimensional parameter, $S_{\alpha} / m b$, or, $x_{c g} / b$

$\bar{T}, \bar{V} \quad=$ instantaneous kinetic and potential energies of the aeroelastic system coupled with an NES

$t, \tau=$ physical and nondimensional times $\left(\tau=\omega_{\alpha} t\right)$

$U \quad=$ constant and uniform flow speed around the wing

$W_{n c}^{y, \alpha} \quad=$ instantaneous nonconservative work done by heave and pitch modes, respectively

$x_{c g}$

$y, v$

$\epsilon$

$\Theta$

$\lambda$

$\mu$

$\xi_{y}, \xi_{\alpha}$

$\Omega$

$=$ location of the center of gravity (c.g.) measured from the e.a. (positive aft of the e.a.)

$=$ nondimensional heave $(y=h / b)$ and NES $(v=z / b)$ modes

$=$ mass ratio between the NES and the wing, $m_{s} / m$

$=$ reduced speed of the flow, $U / b \omega_{\alpha}$

$=$ nondimensional linear viscous damping in the NES, $c_{s} /\left(m_{s} \omega_{\alpha}\right)$

$=$ density ratio, $\rho_{\infty} b S / 2 m$

$=$ nondimensional nonlinear heave and pitch stiffness factors $\left(\xi_{y}=c_{1} b^{2} \Omega^{2}, \xi_{\alpha}=c_{2} r_{\alpha}^{2}\right)$

$=$ frequency ratio, $\omega_{h} / \omega_{\alpha}$ where $\omega_{h}=\sqrt{K_{h} / m}$ and $\omega_{\alpha}=\sqrt{K_{\alpha} / I_{\alpha}}$

\section{Subscripts}

$\begin{array}{ll}\text { ac } & =\text { aerodynamic center } \\ \mathrm{BPC} & =\text { branch point cycle bifurcation } \\ \mathrm{cg} & =\text { center of gravity } \\ \mathrm{d} & =\text { dissipation } \\ \mathrm{ea} & =\text { elastic axis } \\ \mathrm{F} & =\text { flutter } \\ \mathrm{H} & =\text { Hopf bifurcation } \\ \mathrm{LPC} & =\text { limit point cycle bifurcation } \\ \mathrm{NS} & =\text { Neimark-Sacker bifurcation } \\ \mathrm{nc} & =\text { nonconservative } \\ \mathrm{RE} & =\end{array}$

Superscripts

$$
\begin{aligned}
\cdot & =d / d t \\
& =d / d \tau
\end{aligned}
$$

\section{Introduction}

$\mathrm{T}$

HEORETICAL and experimental suppression of aeroelastic instabilities by means of broadband passive

targeted energy transfers was recently studied.1,2 A single degree-of-freedom (DOF) nonlinear energy sink (NES) was coupled to a 2-DOF rigid wing in low-speed subsonic regime with quasi-steady aerodynamic theory (Fig. 1). This nonlinear attachment due to the triggering mechanism of instability ${ }^{3}$ was designed to prevent further energy transfers to the pitch mode from the heave mode at the initial transients.

Lee et al. ${ }^{1}$ also demonstrated that there exist three different types of suppression mechanisms with an SDOF NES; that is, recurring burst-outs and suppression, intermediate, and complete suppression of instability. The recurrent burst-outs of instability was proved to be a critical mechanism for aeroelastic instability caused by a Neimark-Sacker bifurcation of a limit cycle oscillation (LCO).

On the other hand, by performing numerical parametric bifurcation analysis, robustness behavior of each instability suppression was examined. Unless the NES attachment is at the elastic axis, most of all LCO branches tend to possess two limit point cycle (LPC) bifurcation points - one (named, $\Theta_{\mathrm{LPC} 1}$ ) is found at the higher reduced velocity than the Hopf bifurcation point $\left(\Theta_{\mathrm{H}}\right)$, and the other (named, $\Theta_{\mathrm{LPC} 2}$ ), at the lower reduced velocity. It turned out that the relative location of $\Theta_{\mathrm{LPC} 2}$ to $\Theta_{H}$ is crucial to achieve robustness of instability suppression; that is, if the higher $\Theta_{\mathrm{LPC} 2}$ is than $\Theta_{H}$, the better robustness of suppression (regardless of its mechanisms) can be guaranteed.

Figure 2 (a) shows a typical bifurcation diagram when an SDOF NES is attached to a wing. One can first see that the NES makes occurrence of an LCO be shifted to the higher reduced velocity than the linear flutter speed, $\Theta_{F}$. Attaching the NES close to the leading (trailing) edge of the wing tends to locate $\Theta_{\text {LPC2 }}$ at the reduced velocity lower (higher) than $\Theta_{\mathrm{H}}$ (or even lower than $\Theta_{\mathrm{F}}$ ). This proves that applying the 


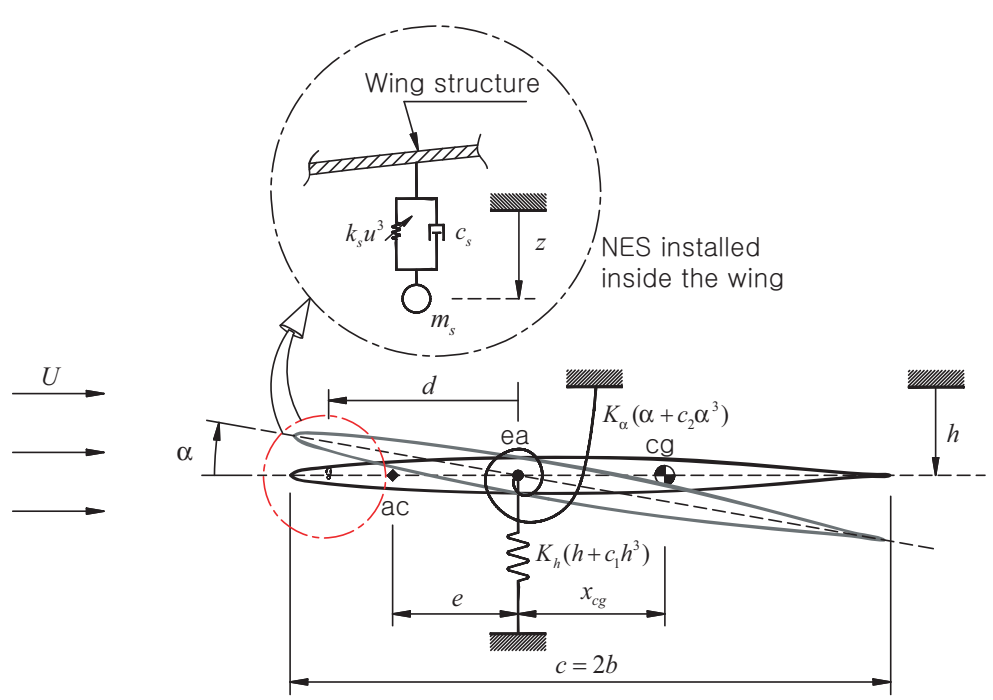

Figure 1. 2-DOF rigid wing model coupled to an SDOF NES.

NES with negative offset (i.e., close to the trailing edge of a wing) generally provides better robustness of instability suppression.

In Fig. 2 (b), one numerical simulation is presented at the reduced speed slightly higher than the Neimark-Sacker bifurcation point $(\Theta=0.915)$ where there can exist one unstable trivial equilibrium, one unstable quasiperiodic LCO responsible for the burst-outing instability, one unstable LCO, and one stable LCO possessing a smaller amplitude than the original LCO. For small initial conditions, the steady state exhibits recurring burst-outs and suppression of instability; however, if a strong disturbance is imposed, then the steady state will be attracted to another stable position, involving a jump to a higher (stable) LCO branch.

In spite of the possibility to obtain robust instability suppression with the SDOF NES, one can still raise an issue about enhancing robustness. In order to address this problem, we introduce a multi-DOF NES configuration instead of the SDOF NES in the next section. Then, comparing changes in the bifurcation diagram, we explain how robustness can be enhanced by using the MDOF NES. Furthermore, we provide numerical simulations to demonstrate robustness enhancement by computing wavelet transforms and modal energy exchanges. Finally, conclusions will be addressed.

\section{Enhancing Robustness of Suppression with MDOF NESs}

In order to improve robustness of instability suppression compared to an SDOF NES, we consider an MDOF NES configuration, shown in Fig. 3. This kind of MDOF NESs in series coupling was studied in Tsakirtzis et al., ${ }^{4}$ basically showing that application of the MDOF NESs can induce even richer dynamics on the frequency-energy plot (FEP). ${ }^{5}$ This means, in turn, that there exists higher possibility for a system to possess more 'baits' to seize the dynamics caused by any disturbances onto a branch in the FEP, making it stick to (or, at least, very close to) the branch until a significant amount of energy is dissipated by a damper in the process of resonance captures.

The MDOF NES is composed of the three equal masses, which in total make the same mass ratio as the SDOF NES. The first mass of the MDOF NES is coupled to a wing only through a linear stiffness, and its main role is a 'bridge' for efficient energy transfers from the wing to the other nonlinear attachments. The second and third ones coupled through essentially nonlinear stiffnesses. The best performance is achieved when the order of the essential nonlinearity between the second and third masses is much smaller than than between the first and second masses; the former is set 50 times less than the latter. 


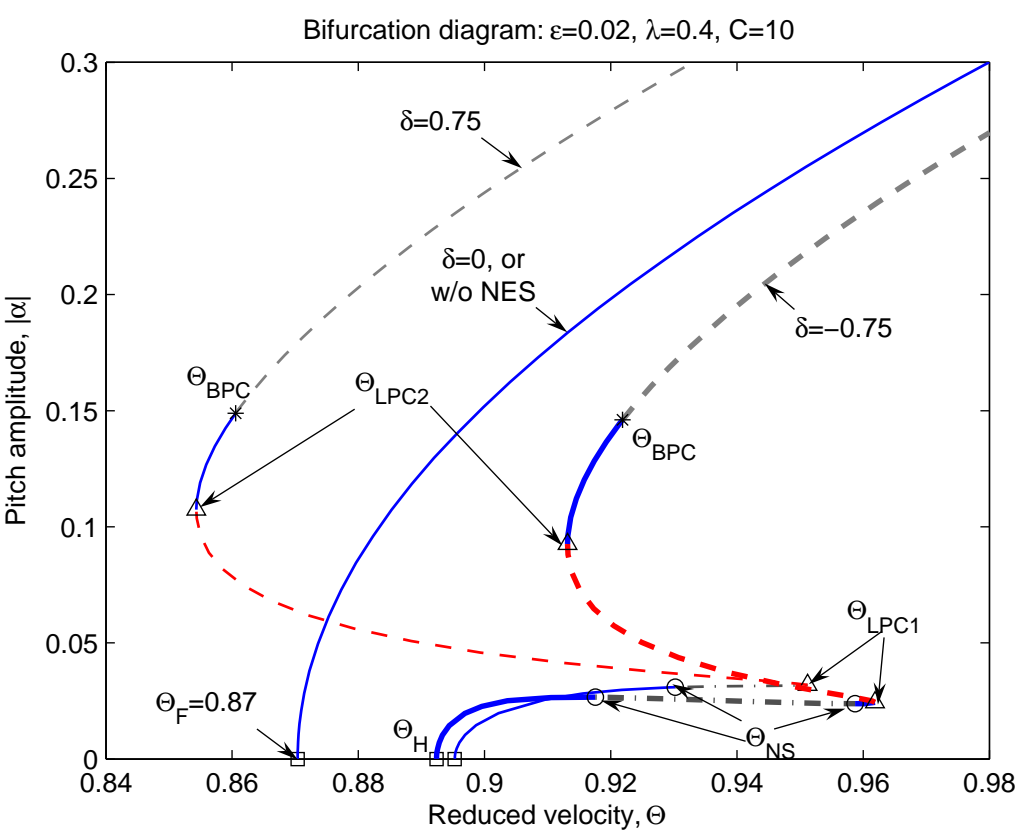

(a)

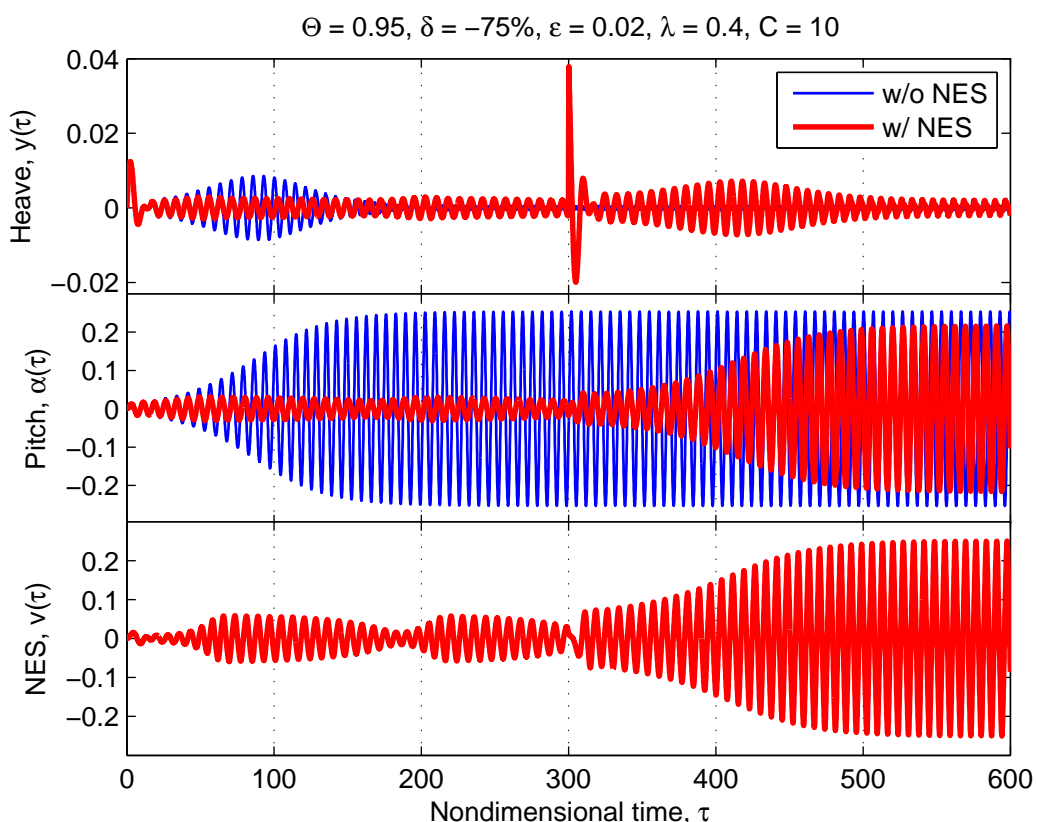

(b)

Figure 2. (a)Bifurcation diagram (incomplete) with respect to $\Theta$ for the SDOF NES $(\epsilon=0.02, \lambda=0.4, C=10)$. Dashed lines indicate unstable LCO branches. Squares (circles, triangles, asterisks) indicate Hopf (NeimarkSacker, limit point of cycles or saddle-node, branch point of cycles) bifurcation points; (b) demonstration of jump phenomenon due to the presence of an impulsive disturbance at $\Theta=0.915$ (i.e., non-robust suppression of instability) between LCO branches due to an impulsive disturbance. All zero initial conditions but for $y /(0)$, and then $20 \times y \prime(0)$ was imposed at $\tau=200$. 


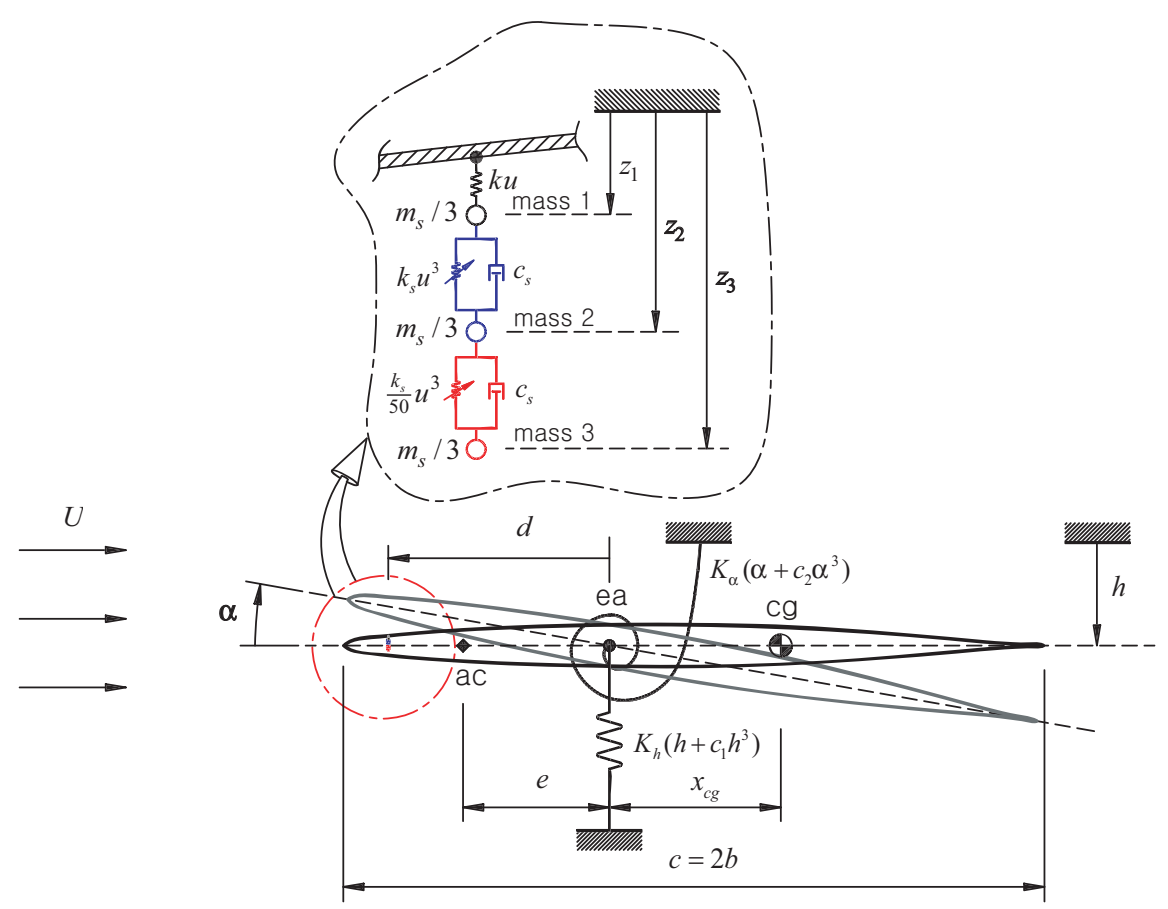

Figure 3. Application of an MDOF NES in series coupling for the 2-DOF rigid wing.

The nondimensional equations of motion can be written:

$$
\begin{aligned}
& y^{\prime \prime}+x_{\alpha} \alpha^{\prime \prime}+\Omega^{2} y+\xi_{y} y^{3}+\mu C_{L, \alpha} \Theta\left(y^{\prime}+\Theta \alpha\right)+C_{1}\left(y-\delta \alpha-v_{1}\right)=0 \\
& r_{\alpha}^{2} \alpha^{\prime \prime}+x_{\alpha} y^{\prime \prime}+r_{\alpha}^{2} \alpha+\xi_{\alpha} \alpha^{3}-\gamma \mu C_{L, \alpha} \Theta\left(y^{\prime}+\Theta \alpha\right)+\delta C_{1}\left(\delta \alpha+v_{1}-y\right)=0 \\
& \frac{1}{3} \epsilon v_{1}^{\prime \prime}+\epsilon \lambda\left(v_{1}^{\prime}-v_{2}^{\prime}\right)+C_{1}\left(v_{1}+\delta \alpha-y\right)+C\left(v_{1}-v_{2}\right)^{3}=0 \\
& \frac{1}{3} \epsilon v_{2}^{\prime \prime}+\epsilon \lambda\left(v_{2}^{\prime}-v_{1}^{\prime}\right)+\epsilon \lambda\left(v_{2}^{\prime}-v_{3}^{\prime}\right)+C\left(v_{2}-v_{1}\right)^{3}+\frac{1}{50} C\left(v_{2}-v_{3}\right)^{3}=0 \\
& \frac{1}{3} \epsilon v_{3}^{\prime \prime}+\epsilon \lambda\left(v_{3}^{\prime}-v_{2}^{\prime}\right)+\frac{1}{50} C\left(v_{3}-v_{2}\right)^{3}=0
\end{aligned}
$$

Then, numerical bifurcation analysis by means of MatCont ${ }^{6}$ is performed to examine changes in LCO branches. A typical result is depicted in Fig. 4. For the purpose of comparison, the LCO branches without an NES and with an SDOF NES are superimposed. The coefficients of the damping and essential nonlinearity are the same for both NES configurations; the mass ratios $(\epsilon)$ for the SDOF and MDOF NESs are 0.02 and 0.014 , respectively. The offset attachments $(\delta)$ for both are selected as \pm 1 .

It can easily be noted that better suppression, as well as its robustness, can be achieved with the MDOF NES of even a smaller mass ratio. Compared to the location of $\Theta_{\mathrm{LPC} 2}$ in Fig. 2 (a) which is slightly above 0.91, the MDOF NES can shift this point near or above 1.0. That is, not only does the MDOF NES enhance robustness of instability suppression, but it can also resist the occurrence of LCOs up to a higher flow speed regime. Robustness enhancement at a relatively high reduced velocity is demonstrated in Fig. 5, where the mass ratio of $2 \%(1.4 \%)$ is considered for the SDOF (MDOF) NES. The aeroelastic system controlled by the MDOF NES exhibits robustness against the impulsive disturbance to the heave mode at the steady state (Fig. 5 (b)), whereas there exists a transition from lower-amplitude unstable quasi-periodic LCO branch to higher-amplitude stable stable LCO branch for the control only with the SDOF NES (Fig. 5 (a)).

\section{Targeted Energy Transfers (TETs)}

We now investigate the TET mechanisms in the case of MDOF NESs. As a first step, wavelet transforms (WTs) are performed to examine the instantaneous frequency behaviors (i.e., transient resonant interactions between the aeroelastic and NES modes), as are summarize in the following.

The WT involves a windowing technique with variable-sized regions so that it performs a multi-resolution analysis, in contrast to the (fast) Fourier transform (FFT) which assumes signal stationarity. Small time 


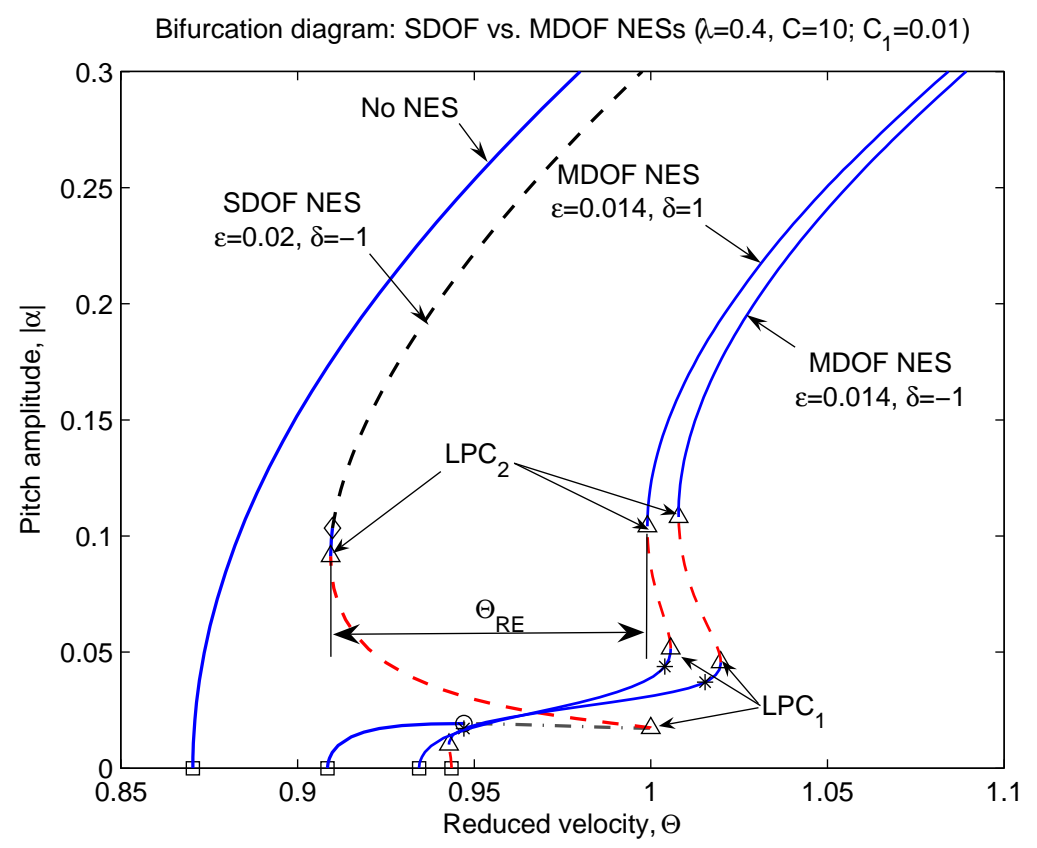

Figure 4. Comparison of bifurcation diagrams: SDOF versus MDOF NESs $\left(\lambda=0.4, C=10, C_{1}=0.01\right)$. The SDOF NES is considered with the mass ratio $\epsilon=0.02$ and the offset $\delta=-1$. The MDOF NES is equipped with $\epsilon=0.014, \delta= \pm 1$. Dashed lines indicate unstable LCO branches. Squares (circles, triangles, diamond, asterisks) indicate Hopf (Neimark-Sacker, limit point of cycles or saddle-node, branch point of cycles, neutral-saddle) bifurcation points. $\Theta_{R E}$ denotes the amount of the enhanced reduced speed for robustness.

intervals are considered for high-frequency components whereas the size of the interval is increased for lowerfrequency components, thereby giving better time and frequency resolutions than the FFT. The Matlab ${ }^{\circledR}$ codes used for the WT computations in this work were developed at the Université de Liège (Liège, Belgium) by Dr. V. Lenaerts in collaboration with Dr. P. Argoul from the Ecole Nationale des Ponts et Chaussées (Paris, France). The Morlet wavelet, $\psi_{M}(\tau)=\mathrm{e}^{-\tau^{2} / 2} \mathrm{e}^{j \omega_{0} \tau}$, which is a Gaussian-windowed complex sinusoid of frequency $\omega_{0}$, is considered as a mother wavelet in this study. The frequency $\omega_{0}$ for the Morlet WT is a user-specified parameter which allows one to tune the frequency and time resolutions of the results. The plots represent the amplitude of the WT as a function of frequency (vertical axis) and time (horizontal axis). Heavily shaded areas correspond to regions where the amplitude of the WT is high, whereas lightly shaded regions correspond to low amplitudes. Such plots enable one to deduce the temporal evolution of the dominant frequency components of the signals analyzed. Comparing the instantaneous frequency contents of the aeroelastic and NES modes provides an additional (direct) way to verify the occurrence of resonance captures, or frequency locking in the transient dynamics.

The instantaneous kinetic and potential energies, which are stored in the masses and springs, respectively, can be written:

$$
\begin{aligned}
\bar{T}(\tau) & =\frac{1}{2} y^{\prime}(\tau)^{2}+x_{\alpha} y^{\prime}(\tau) \alpha^{\prime}(\tau)+\frac{1}{2} \alpha^{\prime}(\tau)^{2}+\frac{1}{3} \epsilon\left[v_{1}^{\prime}(\tau)^{2}+v_{2}^{\prime}(\tau)^{2}+v_{3}^{\prime}(\tau)^{2}\right] \\
\bar{V}(\tau) & =\frac{1}{2} \Omega^{2} y(\tau)^{2}+\frac{1}{2} r_{\alpha}^{2} \alpha(\tau)^{2}+\frac{1}{4} \xi_{y} y(\tau)^{4}+\frac{1}{4} \xi_{\alpha} \alpha(\tau)^{4} \\
& +\frac{1}{4} C\left[v_{1}(\tau)-v_{2}(\tau)\right]^{4}+\frac{1}{200} C\left[v_{2}(\tau)-v_{3}(\tau)\right]^{4}+\frac{1}{2} C_{1}\left[y(\tau)-\delta \alpha(\tau)-v_{1}(\tau)\right]^{2}
\end{aligned}
$$

so that the total energy can be computed:

$$
E^{T o t a l}(\tau)=\bar{T}(\tau)+\bar{V}(\tau)
$$

The energy input from the flow, which is a sum of the initial energy provided by the initial conditions and the nonconservative work done by the flow, can be expressed in the following.

$$
E^{\text {Input }}(\tau)=E^{\text {Total }}(0)+W_{n c}^{y}(\tau)+W_{n c}^{\alpha}(\tau)
$$




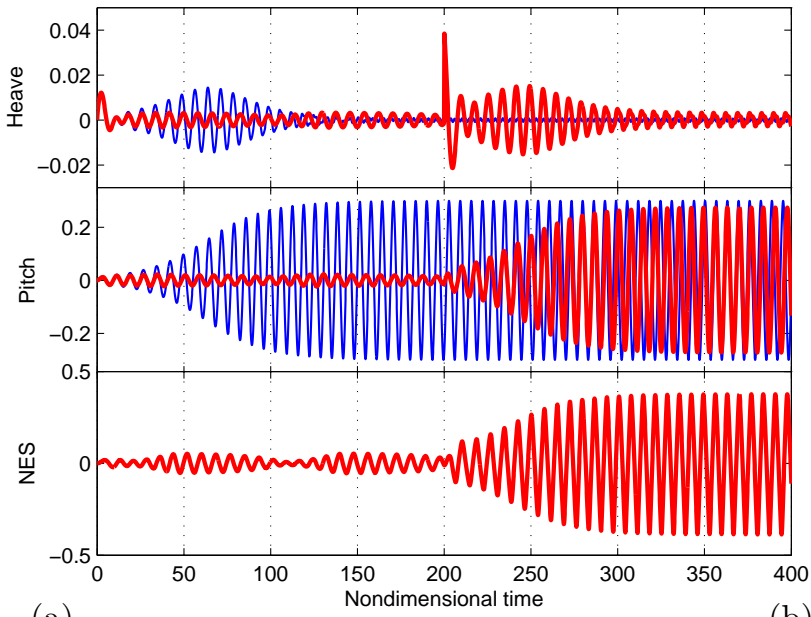

(a)

(b)

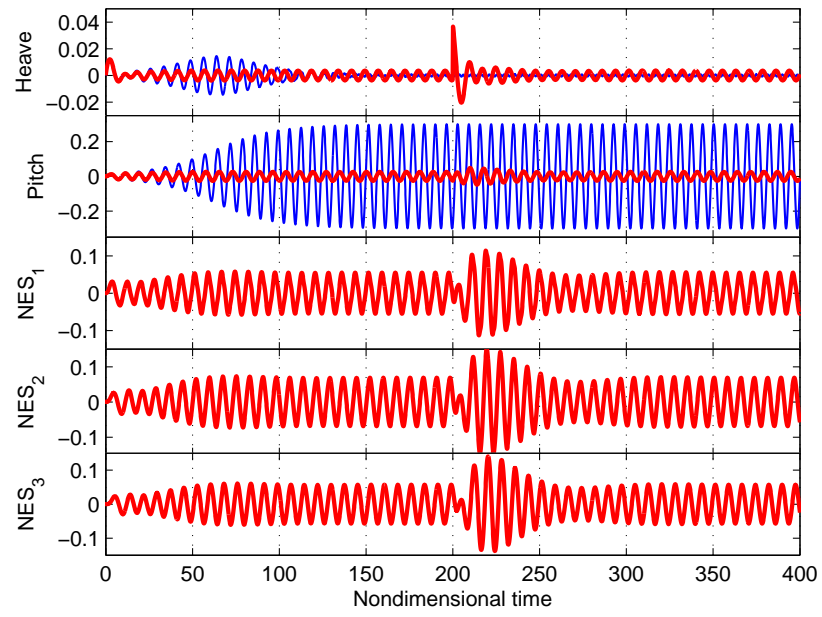

Figure 5. Demonstration of enhancing robust suppression of instability under the impulsive disturbance of $20 \times y^{\prime}(0)$ at $\tau=200$ when $\Theta=0.98, \delta=-1, \lambda=0.4, C=10, C_{1}=0.01$ (a) with an SDOF NES $(\epsilon=0.02)$, and (b) with an MDOF NES $(\epsilon=0.014)$. The thick lines indicate the controlled responses.

where

$$
\begin{aligned}
& W_{n c}^{y}(\tau)=\mu C_{L, \alpha} \Theta \int_{0}^{\tau}\left\{y^{\prime}(s)+\Theta \alpha(s)\right\} y^{\prime}(s) d s \\
& W_{n c}^{\alpha}(\tau)=-\gamma \mu C_{L, \alpha} \Theta \int_{0}^{\tau}\left\{y^{\prime}(s)+\Theta \alpha(s)\right\} \alpha^{\prime}(s) d s
\end{aligned}
$$

The total energy dissipation by the MDOF NES can be written:

$$
E_{d}^{N E S}(\tau)=E_{d}^{N E S 1}(\tau)+E_{d}^{N E S 2}(\tau)
$$

where

$$
E_{d}^{N E S 1}(\tau)=\epsilon \lambda \int_{0}^{\tau}\left[v_{1}^{\prime}(s)-v_{2}^{\prime}(s)\right]^{2} d s, E_{d}^{N E S 2}(\tau)=\epsilon \lambda \int_{0}^{\tau}\left[v_{2}^{\prime}(s)-v_{3}^{\prime}(s)\right]^{2} d s
$$

As a result, the following instantaneous energy balance should hold:

$$
E^{\text {Total }}(\tau)=E^{\text {Input }}(\tau)-E_{d}^{N E S}(\tau)
$$

We set the flow speed as $\Theta=0.92$, for which a complete elimination of aeroelastic instability can be realized (see Fig. 4). For all zero initial conditions except for $y \prime(0)=0.1$, one obtains the responses shown in Fig. 6 (a). Initial transients exhibits beating-like interactions between the aeroelastic and NES modes. One can see, from Fig. 6 (c), that a significant amount of energy is extracted to the MDOF NES masses in the first two big interactions (i.e., $\tau \in[0,100] ; E^{y}, E^{\alpha}, E^{u}, E^{v}$ and $E^{w}$ respectively denote the energies contained the heave and pitch modes, the first, second, and the third of the MDOF NES). Moreover, a balance between the energy input and the energy dissipation by the NES is reached in this period. It should be desirable that more energy dissipation occurs in the second damper (i.e., $E_{d}^{N E S 1}<E_{d}^{N E S 2}$ ), which demonstrates more energy is transferred to the third mass than the second mass. Also, WTs in Fig. 6 (b) proves that there exist strong nonlinear modal interactions involving transient resonance captures ${ }^{3}$ in this period.

Another interesting observation, which is not included in this paper, is that, for a smaller $y \prime(0)$, it takes longer time to reach a steady state (i.e., a balance between the input energy and energy dissipation). Also, the smaller disturbances yield energy transfer mechanism which make the second mass possess the more energy than the third one. Different from the case with a strong $y \prime(0)$, more energy dissipation occurs in the first damper (i.e., $E_{d}^{N E S 1}>E_{d}^{N E S 2}$ ). This observation again proves that more efficient nonlinear energy pumping can be realized for sufficiently large input energy (see, for example, Vakakis and Gendelman ${ }^{7}$ ). The MDOF NESs with a positive offset tend to exhibit less number of initial transient interactions. 

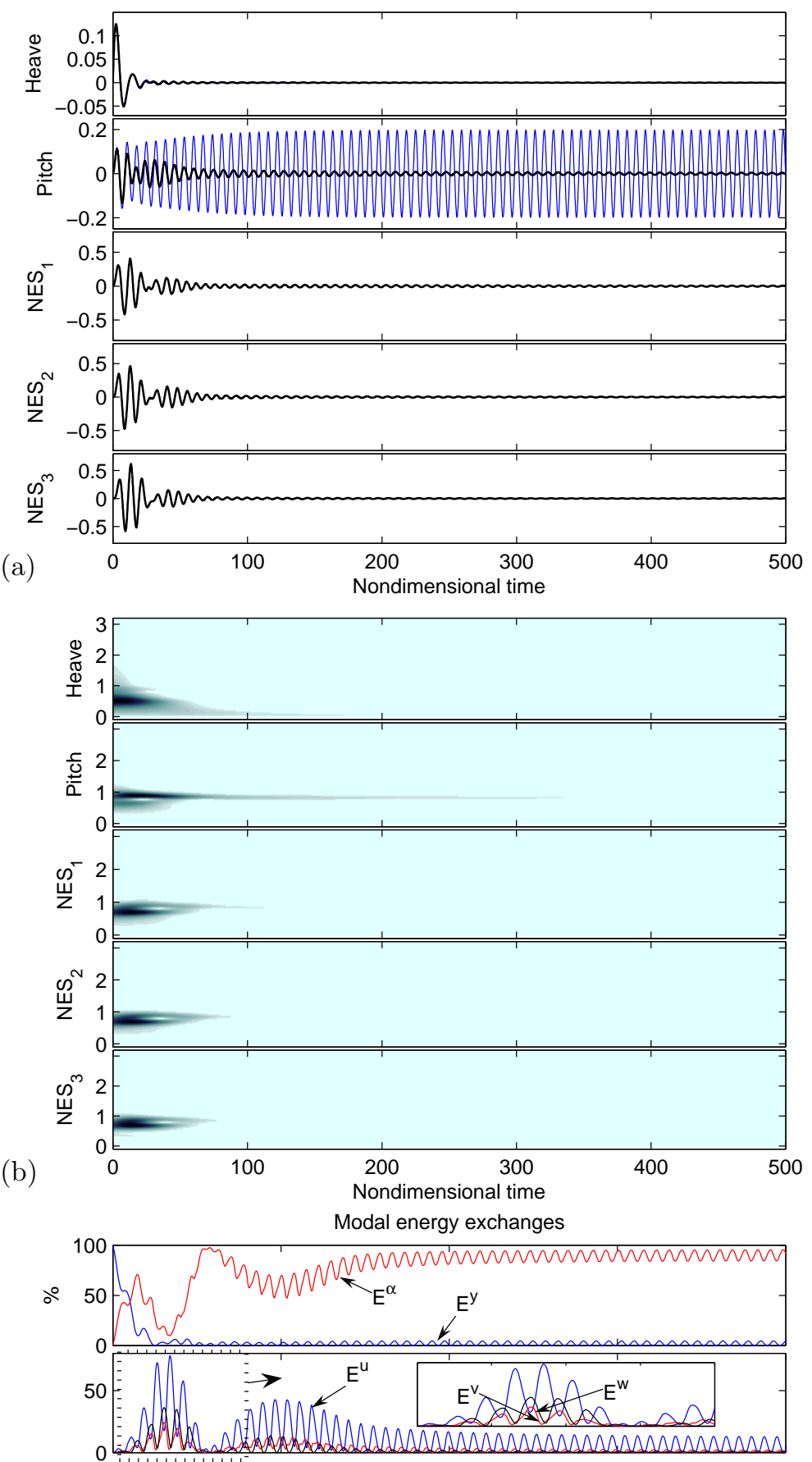

Energy input and dissipation

(c)
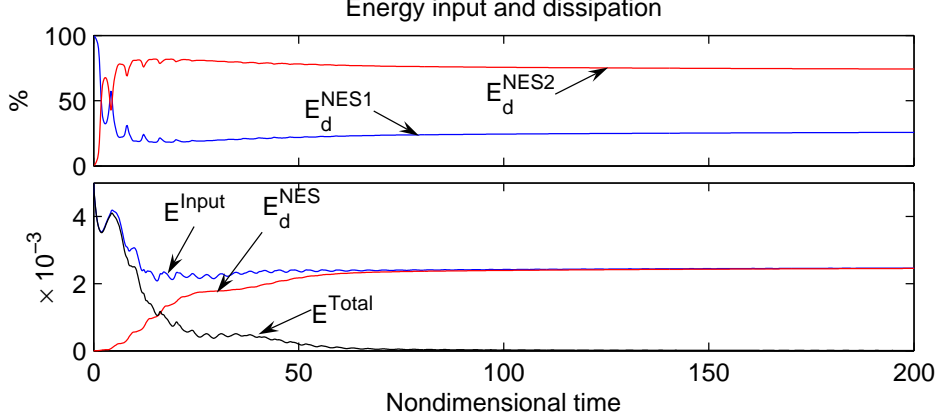

Figure 6. Wing responses when the MDOF NES is applied $\left(\Theta=0.92, \epsilon=0.014, \lambda=0.4, C=10, C_{1}=0.01, \delta=\right.$ $-1, y \prime(0)=0.1)$ : (a) Time series (thin lines for uncontrolled responses); (b) wavelet transforms; (c) energy exchanges between the aeroelastic and NES modes. 


\section{Conclusions}

In order to improve robustness of aeroelastic instability suppression in a rigid wing with structural nonlinearities, we considered a MDOF NES configuration. From the bifurcation analysis of the dynamics of the integrated wing-NES system by means of a numerical continuation technique, we showed that the control of the occurrence of the lower LPC bifurcation point above a Hopf bifurcation point is crucial to enhancing robustness. Moreover, we demonstrated that the proposed MDOF NES design (composed of multiple masses coupled in series by means of essentially nonlinear springs and viscous dampers) does not only greatly enhance the robustness of LCO suppression against even strong impulsive disturbances, but also achieve better or at least comparable suppression performance for smaller total masses compared to SDOF NESs under identical disturbances. Nonlinear modal interactions between the wing and the attached MDOF NES were studied by means of WTs, as well as the resulting modal energy exchanges. The results suggest that an appropriately designed MDOF NES can greatly improve robustness of instability suppression. The results reported in this work, which should be viewed in conjunction with earlier theoretical and experimental results, ${ }^{1,2}$ indicate that appropriately designed lightweight passive nonlinear absorbers with essential stiffness nonlinearities can suppress, effectively and robustly, LCO instabilities. As shown in other work, ${ }^{4}$ the dynamical mechanism governing this suppression is a series of resonance captures that occur between the wing modes and multiple modes of the NES, which result in broadband passive targeted transfer of unwanted vibration energy from the wing to the NES, where this energy is localized and passively dissipated. Additional work is needed to study the complex and highly degenerate structure of the dynamics of the integrated structure-MDOF NES system.

\section{Acknowledgement}

This work was supported by the US Air Force Office of Scientific Research through Grant Number FA9550-04-1-0073. GK is supported by a grant from the Belgian National Science Foundation, which is gratefully acknowledged.

\section{References}

\footnotetext{
${ }^{1}$ Lee, Y., Vakakis, A., Bergman, L., McFarland, D., and Kerschen, G., "Suppressing Aeroelastic Instability Using Broadband Passive Targeted Energy Transfers, Part I: Theory," AIAA Journal, Vol. 45, No. 3, March 2007, pp. 693-711.

${ }^{2}$ Lee, Y., Kerschen, G., McFarland, D., Hill, W., Nichkawde, C., Strganac, T., Bergman, L., and Vakakis, A., "Suppressing Aeroelastic Instability Using Broadband Passive Targeted Energy Transfers, Part II: Experiments," AIAA Journal, in review.

${ }^{3}$ Lee, Y., Vakakis, A., Bergman, L., McFarland, D., and Kerschen, G., "Triggering Mechanisms of Limit Cycle Oscillations in a Two-degree-of-freedom Wing Flutter Model," Journal of Fluids and Structures, Vol. 21, No. 5-7, 2005, pp. 485-529.

${ }^{4}$ Tsakirtzis, S., Panagopoulos, P., Kerschen, G., Gendelman, O., Vakakis, A., and Bergman, L., "Complex Dynamics and Targeted Energy Transfers in Linear Oscillators Coupled to Multi-Degree-of-Freedom Essentially Nonlinear Attachments," Nonlinear Dynamics, Vol. 48, No. 3, May 2007, pp. 285-318.

${ }^{5}$ Lee, Y., Kerschen, G., Vakakis, A., Panagopoulos, P., Bergman, L., and McFarland, D., "Complicated Dynamics of a Linear Oscillator with a Light, Essentially Nonlinear Attachment," Physica D, Vol. 204, No. 1-2, 2005, pp. 41-69.

${ }^{6}$ Dhooge, A., Govaerts, W., and Kuznetsov, Y., "MATCONT: A Matlab Package for Numerical Bifurcation Analysis of ODEs," ACM Transactions on Mathematical Software, Vol. 29, No. 2, 2003, pp. 141-164.

${ }^{7}$ Vakakis, A. and Gendelman, O., "Energy Pumping in Coupled Mechanical Oscillators, Part II: Resonance Capture," Transactions of the ASME, Journal of Applied Mechanics, Vol. 68, January 2001, pp. 42-48.
} 